\section{rev Psi}

Revista de Psicología (UNLP)

https://revistas.unlp.edu.ar/revpsi

\title{
Maternidad cuestionada: diferencias sobre las creencias hacia la maternidad en mujeres
}

\author{
Nicole Obregón Brocher ${ }^{1}$ \\ Daniela Arari Zayat ${ }^{1}$ \\ Correspondencia \\ carolina.armenta@ibero.mx
}

Filiaciones institucionales

${ }^{1}$ Universidad Iberoamericana (México)

\author{
Carolina Armenta Hurtarte ${ }^{1}$ \\ Regina Ortiz-Izquierdo Herington ${ }^{1}$
}




\section{Maternidade questionada: diferenças sobre crenças em relação à maternidade em mulheres}

\section{Resumo}

Do ponto de vista sociocultural, a maternidade é considerada uma característica natural da mulher e foi identificada como um objetivo de vida e realização. No entanto, essa construção social foi transformada ao longo do tempo por fatores sociais e políticos que são possíveis de identificar em programas sociais que promovem o empoderamento das mulheres e sua participação em outros campos sociais, a fim de melhorar sua desvantagem social. Portanto, o objetivo deste estudo foi avaliar se existe um efeito de empoderamento e autoconceito nas crenças sobre a maternidade como um dever social e senso de vida. Os resultados confirmam que há realmente um efeito e, mesmo foi possível identificar diferenças em mulheres que desejam maternidade. Esses achados permitem questionar o efeito da socialização cultural como enculturação na construção de um autoconceito pessoal e na reinterpretação pessoal como mãe.

\section{Palavras-chave}

maternidade $\mid$ cultura $\mid$ crencas $\mid$ atitudes $\mid$ empoderamento

\section{Questioned motherhood: Differences about beliefs towards motherhood in women}

\section{Abstract}

Motherhood is considered as a natural feature of women and has been identified as a goal of life and developmental achievement. However, this social construction has been transformed over time by social and political factors that are possible to identify in social programs that promote empowerment of women and their participation in other social fields in order to improve their social disadvantage. Therefore, the purpose of this study was to assess whether there is an effect of empowerment and self-concept on beliefs about motherhood as a social duty and a sense of life. The results confirm that there is indeed an effect and, even it was possible to identify differences in women who want motherhood. These findings allow us to question the effect of cultural socialization as enculturation on a personal self-concept construction and personal reinterpretation as mothers.

\section{Keywords}

motherhood | culture | beliefs | attitudes | empowerment 


\section{Aspectos destacados del trabajo}

- Existe un efecto del autoconcepto en las creencias sobre la maternidad en mujeres.

- El autoconcepto es diferente entre mujeres quienes desean ser madres de quienes no lo desean.

- Mujeres que desean ser madres tienen un mayor empoderamiento que se refiere a participación social.

- Quienes tienen autoconcepto depresivo tienen creencias sobre maternidad como deber social y sentido de vida.

Durante mucho tiempo se ha tenido la creencia de que la mujer debe ser madre para realizarse y se ha visto como la meta final de ser mujer. Esta idea se debe a que gran parte de las representaciones y significados sociales de las mujeres se representa a través de la maternidad (Langer, 2002). A lo largo de la historia, las creencias acerca de la maternidad han ido cambiando debido a factores culturales y sociales (Mota, Calleja, Sánchez, y Carreño, 2018). En México se ha favorecido estas creencias mediante dichos que se socializan desde la familia y se mantienen entre las amistades así como en medios de comunicación, siendo evidentes incluso en los comerciales de venta y promocionales; estás frases que se pueden encontrar son (Rocha y Díaz, 2005): "una mujer se realiza hasta que es madre", "lo más importante para una mujer es ser madre", "lo que más desea una mujer es tener uno o más hijos", "una mujer es más feliz si es madre", "para una mujer, ningún logro se compara con ser madre", "para sentirse feliz, una mujer necesita tener un hijo", "una mujer está completa hasta que es madre", "una mujer se realiza hasta que tiene un hijo", "la vida vale la pena si tienes hijos", "si una mujer no tiene hijos, merece el rechazo de los demás”, entre otras (Mota et al. 2018).

A pesar de que estas creencias son elementos esenciales para un contexto cultural que determina las interacciones y conductas esperadas socialmente e incluso permean el autoconcepto que se conforma, en este caso de las mujeres, es fundamental indicar que existen factores que permean su cambio y se favorecen otras construcciones individuales. Estos cambios se pueden identificar mediante programas sociales desarrollados que buscan generar la equidad y eliminar la violencia de género en el contexto mexicano que incluso ha llevado a generar empoderamiento de las mujeres (INM, 2019). El empoderamiento femenino surge a partir del movimiento social de las mujeres por las diferencias de género impuestas por la sociedad a lo largo de la historia y, desde el enfoque feminista, este incluye tanto el cambio individual como la acción colectiva para generar una alteración de las estructuras y procesos que reproducen la posición subordinación en las mujeres como es el sistema de género (Bethencourt y Carrillo, 2014). 
En este sentido, para que ocurra el cambio individual, Rodríguez (2018) menciona que hay que centrarse en los factores intrínsecos del empoderamiento como la autoconfianza y la fuerza interna pues son fundamentales para tomar control sobre los propios recursos y definirse a sí mismas al tomar una posición determinada en la sociedad. A partir de estas definiciones, es necesario indicar que el empoderamiento debe ser un concepto que integre, tanto la capacidad de incidencia que se tiene sobre el contexto, como las capacidades individuales de cada mujer como la autoconfianza y seguridad para darle a su vida la dirección que ellas deseen, siendo estos elementos del autoconcepto.

El autoconcepto, hasta los años sesenta, se mantuvo como una concepción unidimensional, siendo definido como una visión global que las personas construían de sí mismas (Kimble et al., 2002). Sin embargo, una profunda revisión de lo hasta entonces investigado puso de manifiesto la carencia y pobreza de los parámetros ejercidos para evaluarlo (Burns, 1979), lo que abrió paso a nuevas propuestas sobre un abordaje más integral del mismo. Una de las proposiciones más destacables fue elaborada por Shavelson, Hubner y Stanton (1976) quienes abordan el autoconcepto desde una perspectiva multidimensional en la que incluyen distintos aspectos de la persona, de modo que está compuesto por el autoconcepto académico y el no académico; el segundo, incluye el autoconcepto social, emocional y físico. El autoconcepto académico ha ido conquistando la atención de la psicología debido a su estrecha relación con el desarrollo y desempeño educativo, sin embargo, para el propósito de este trabajo, se integrará al autoconcepto no académico.

Como se indicó anteriormente, el autoconcepto no académico está compuesto por el autoconcepto social, emocional y físico. El autoconcepto físico se compone por dos dimensiones: la apariencia física y la habilidad física. Mientras que el autoconcepto emocional se refiere a la inteligencia emocional que posee la persona y la capacidad para regular las propias emociones (Esnaola, Goñi y Madariaga, 2008). Finalmente, el autoconcepto social se refiere a la diferenciación entre pares, así como a las habilidades para formar relaciones positivas (González y Goñi, 2005). A pesar de que no existe un consenso único referente al número e identidad de los dominios como de los subdominios del autoconcepto, la multidimensionalidad de este queda corroborada por los datos de múltiples investigaciones (Harter, 1990).

El autoconcepto está en constante evolución, pues se va modificando a través de la experiencia (Zabala y Fernández, 2005) por lo que es necesario realizar una evaluación que integre distintas dimensiones. Ante esto, por autoconcepto se puede comprender el conjunto de percepciones que tiene el individuo sobre sí mismo (Harter, 1990), así como una formación cognoscitiva que se va forjando a partir de una mezcla de imágenes de lo que somos, de lo que deseamos ser y de lo que manifestamos a los demás (González y Tourón, 1992). Por lo tanto, el rol de la maternidad al considerarse una imagen que se manifiesta socialmente está asociada con el autoconcepto de las mujeres, afectando sus percepciones individuales y la formación de su propia identidad. 
El rol de la maternidad se ha naturalizado socialmente. Se ha enfatizado la parte biológica como un papel importante de la mujer dado que la procreación es un proceso orgánico (Anzaldúa, 2004). Por lo tanto, se ha generado la creencia de que la maternidad en sí es, igualmente, un deseo innato de las mujeres, restando importancia a la influencia que tiene la sociedad y la cultura con respecto a esta creencia (Ávila, 2005). Integrando la mirada cultural desde el género, se puede entender que la maternidad obligatoria ha sido impuesta por el contexto social pues a lo largo del tiempo, las mujeres han sido definidas a partir de su capacidad de procrear y se han visto enmarcadas por la parte doméstica y de crianza de los hijos e hijas, reduciendo su significado personal a ser madres y esposas (Castillo, 2008).

Los factores histórico-socioculturales han generado una simbiosis entre ser mujer y ser madre ya que, a partir de las creencias, se ha determinado a la maternidad como una característica esencial de la mujer, que la define convirtiéndose en una meta que deben lograr (Valverde y Cubero, 2014). Esto ha originado múltiples consecuencias como imponer la maternidad como obligatoria, así como limitar las oportunidades de las mujeres para un desarrollo en el ámbito laboral y social y, a la vez, limitar su capacidad para tomar el control sobre su vida (Castillo, 2008).

A partir de los cuestionamientos generados al sistema de creencias, se ha permeado la idea sobre la maternidad como una decisión propia y no una imposición por parte de las creencias sociales o familiares. La presión económica de los años 70, la política mundial, los movimientos feministas de la segunda ola con acciones y consignas como "dueñas de nuestros cuerpos, dueñas de nuestras vidas" así como el empoderamiento femenino han cuestionado los roles de género lo cual, ha permitido crear nuevas identidades para la mujer y modificar sus prioridades (Ávila, 2005). Esta reflexión ha permitido que varias mujeres se cuestionen las creencias sobre la maternidad desviándose de los roles impuestos tradicionalmente.

Hoy en día, las mujeres empiezan a cuestionarse los factores que las llevan a considerar el ser madre como una característica obligatoria de su género, empiezan a reflexionar sobre su vida, sus habilidades, necesidades e intereses y buscan responder también a las demandas económicas y sociales del sistema actual como entrar al campo laboral y profesional. De acuerdo con Castillo (2008) estos cambios permiten reflexionar sobre la importancia de las carreras y las profesiones para las mujeres y cómo ha impactado en su cuestionamiento sobre la maternidad. El interés de las mujeres en el campo laboral y profesional ha generado el reconocimiento por la necesidad de la incorporación de los hombres en el cuidado de las/os hijas/os, transformando el concepto de la maternidad como una actividad exclusiva de las mujeres y buscar la integración de la paternidad en la crianza.

Se ha observado que para conocer a fondo las creencias sociales que enfrentan las mujeres ante la normatividad de la maternidad es necesario analizar el lenguaje, las leyes políticas, los aspectos sociales y el tratamiento médico de las mujeres. De acuerdo con Daniluk (1999), las mujeres sin hijas/os son comúnmente asociadas con el egoísmo, la inmadurez, la frialdad y la insuficiencia por lo que genera una 
connotación negativa socialmente. Por otro lado, en el aspecto clínico, aún se mantiene la creencia de que la maternidad actúa como un método de sanación para malestares físicos como los trastornos menstruales, alcanzar la madurez emocional y psíquica en la maternidad (Daniluk, 1999).

De igual forma, socialmente se puede percibir el fuerte impacto de la religión y de grupos que buscan mantener la conformación tradicional de la familia en la que se enfatiza el rol tradicional de las mujeres como madres (Ávila, 2005). Organizaciones como Frente Nacional por la Familia (2019) han realizado movimientos sociales para mantener las creencias, expectativas y normatividades sociales de las mujeres en la maternidad como meta final. Sin embargo, se ha desarrollado una demanda para reanalizar la configuración de la maternidad en la modernidad para integrar las intersecciones que permean y demanda el reconocimiento de los derechos de las mujeres quienes son madres (Sánchez, 2016).

Desafortunadamente, la mirada tradicional sobre la maternidad tiene múltiples consecuencias, especialmente en cómo se vive la maternidad en aquellas mujeres en las que no fue deseada y el impacto que esto tiene el los/as hijos/as que cría. De acuerdo con Yanina Ávila (2003), las experiencias y presiones sociales a las que se encuentran expuestas las mujeres al cumplir con el destino de ser madres influyen de forma negativa sobre las mujeres a partir de la estigmatización. Investigaciones hechas por Loeber (1986) y por Sampson (1993) evidencian el impacto de una maternidad obligatoria en la que existe falta de supervisión y trato hostil los cuales, contribuyen a una conducta violenta que puede llevar a la delincuencia juvenil. Asimismo, Rasanen y colaboradores (1991) demostraron que la maternidad adolescente vinculada con bajo nivel académico y el hecho de que el embarazo no fuese deseado, aumentan las posibilidades de conductas agresivas. De acuerdo con Ana Langer (2002), los/as hijos/as no deseados/as se han desarrollado en un ambiente de violencia y abandono. Estas evidencias permiten identificar que la maternidad vista como una obligación tiene consecuencias adversas tanto en la madre como en las hijas/os que tenga por lo que es necesario mantener la maternidad como una opción y no una obligación social como meta final de las mujeres, de igual manera es necesario que a la maternidad y la crianza se les reconozcan su valor económico, social y político que tiene para reconstruir la imagen de esta (Vivas, 2019).

Los índices de natalidad en México permiten identificar la situación en la que se encuentran las mujeres al momento de dar a luz. En primera instancia es posible identificar que los índices de natalidad anuales que se reportan en México (INEGI, 2019) han decrecido, siendo el actual de 2 hijas/os por mujer. En 2017, se identificó que, a pesar de presentarse una disminución en la natalidad nacional, aún tres cuartas partes de las mujeres han sido madres en algún momento de su vida. Asimismo, se observó que la tasa global de fecundidad es distinta a partir del grado académico que tienen las mujeres en donde se encuentra que a mayor educación disminuye la tasa de fecundidad. Finalmente, se observó que cuando las mujeres tienen mayor número de hijas e hijos su participación en el ámbito laboral fuera del hogar se disminuye 


\section{(INMUJERES, 2019).}

Es posible observar que la presencia de hijas o hijos tiene una relación con el ejercicio laboral y el nivel salarial que perciben las mujeres, lo cual genera inequidad. Por lo tanto, se han realizado acciones gubernamentales dirigidos a eliminar esta situación mediante programas sociales que buscan la equidad a partir de la promoción del empoderamiento y la reconstrucción del autoconcepto de la mujer. Estas acciones sociales pueden permear sus creencias hacía la maternidad, por lo que el objetivo de este estudio es identificar la influencia que tiene la percepción empoderamiento y autoconcepto en sus creencias sobre la maternidad.

\section{Método}

\section{Muestra}

Para los fines de esta investigación se conformó una muestra no probabilística por conveniencia que se conformó por 296 mujeres con un rango de edad entre 18 a 29 años con una media de 22 años y desviación estándar de 2.8 años, de las cuales $89.52 \%$ no son madres. El grado escolar de las participantes fue de $3.6 \%$ en secundaria, $17.8 \%$ en preparatoria, $76.2 \%$ en licenciatura, $0.3 \%$ en una especialidad y $0.3 \%$ en una maestría, las demás participantes no desearon informar. De dicha muestra el 51.5\% tiene un trabajo remunerado de las cuales, el $28.7 \%$ trabajan de medio tiempo y el $22.8 \%$ de tiempo completo; mientras que el $48.3 \%$ reportó no tener un trabajo económicamente remunerado. Asimismo, el 36.6\% considera que su trabajo está adecuadamente remunerado, mientras que el 14.5\% considera que no está adecuadamente compensada. De las participantes al momento de la aplicación reporta el $54.8 \%$ que tiene una pareja romántica mientras que el $44.2 \%$ no tiene una relación amorosa, las demás no desearon informar. Finalmente, el 37.0\% de las mujeres consideran la adopción como una posibilidad de la maternidad, mientras que el $40.9 \%$ no lo considera como una opción; las demás mujeres no desearon responder. Asimismo, el 63\% de las mujeres planean tener alguna hija o hijo, mientras que el $15.2 \%$ no lo planean.

\section{Instrumentos}

Para cumplir con el objetivo de este estudio se aplicaron tres diferentes instrumentos a la muestra con la finalidad de explorar el empoderamiento, el autoconcepto y las creencias sobre la maternidad, así como un cuestionario sociodemográfico. La primera escala que se aplicó fue la Escala de Creencias sobre la Maternidad (González, Calleja, y Meléndez, 2018), la cual está conformada por 13 reactivos con respuesta tipo Likert de (1) que equivale a totalmente en desacuerdo a (5) que refiere a totalmente de acuerdo y tiene una Confiabilidad Alpha de Cronbach de 0.92. La escala se encuentra dividida en dos dimensiones: la maternidad como sentido de vida mediante 8 reactivos con un $\alpha=.917$ (por ejemplo: "lo más importante para una mujer es ser madre", "lo más importante para una mujer es tener uno o más hijos", 
"una mujer es más feliz si es madre") y la maternidad como deber social evaluada mediante 5 reactivos con confiablidad alfa de 0.831 (por ejemplo: "si una mujer no tiene hijos merece el rechazo de los demás", "para una mujer vale la pena vivir solo si tiene hijos", "el valor de una mujer depende de que sea madre").

Para la evaluación del autoconcepto, se utilizó la escala de Autoconcepto de DíazLoving, Reyes-Lagunes y Riviera-Argón (2002). Está compuesta por 48 reactivos con respuesta tipo Likert de cinco puntos (1. Totalmente de acuerdo a 5. Totalmente en desacuerdo) y tiene una confiablidad Alpha de Cronbach de $\alpha=.838$. Los factores de la escala son: social-expresivo (por ejemplo: alegre, divertida, animada, amigable, sociable, simpática), inteligencia socioemocional (por ejemplo: tranquila, serena, calmada, pacífica, relajada), ético-normativo (por ejemplo: honrada, honesta, leal, respetuosa), social-afiliativo (por ejemplo: romántica, tierna, sentimental, amorosa, cariñosa), depresión (por ejemplo: melancólica, nerviosa, ansiosa, triste), instrumental-constructivo (por ejemplo: activas, cumplidas, ordenadas, y trabajadoras) y la vulnerabilidad emocional (por ejemplo: amargada, apática, solitaria, tímida, voluble).

Para medir el empoderamiento, se utilizó la escala de Agencia Personal y Empoderamiento (ESAGE) (Pick et al., 2007). La escala se encuentra dividida en dos dimensiones: Agencia Personal, la cual se refiere a una falta de confianza en sí mismas e inseguridad mediante 35 reactivos como: "me es difícil expresar mi opinión públicamente" y tiene una confiablidad de $\alpha=.718$; y Empoderamiento, el cual se refiere a la percepción de la persona sobre la incidencia que tiene sobre su contexto mediante 7 reactivos (por ejemplo: en mi colonia/comunidad ayudo a resolver los conflictos que se presentan) y tiene una confiablidad de $\alpha=.749$.

\section{Procedimiento}

La aplicación de los instrumentos se realizó en lugares públicos y privados, buscando una población de sexo femenino con un rango de edad entre 18 a 29 años para tener información de la etapa de juventud mexicana. Los lugares públicos concurridos fueron centros, plazas, establecimientos y parques. Por otra parte, los lugares privados fueron, universidades y hogares, donde se reclutará a las participantes. La aplicación se realizó de manera individual en donde se les aseguró la confidencialidad y el anonimato de las participantes y se les aseguró que se utilizarán para fines estadísticos; asimismo, se les resolvieron aquellas dudas que tuvieron durante la aplicación y se les informó que podrían suspender la aplicación en el momento que lo desearan.

\section{Diseño de investigación}

Para cumplir con el objetivo de esta investigación se realizó un diseño no experimental transversal mediante encuesta con un alcance correlacional-explicativo. 


\section{Análisis de datos}

A partir de la información obtenida se realizaron los análisis en el programa SPSS v.25. en primera instancia se realizó una prueba $t$ de Student para comparar el empoderamiento, autoconcepto y creencias sobre la maternidad entre mujeres que desean ser madres y quienes no; así como entre mujeres que son madres y quienes no. Posteriormente, se llevó a cabo una correlación de Pearson para identificar la asociación entre las variables de empoderamiento, autoconcepto y creencias sobre la maternidad. Finalmente, se efectuó un análisis de regresión en donde el empoderamiento y el autoconcepto son las variables de entrada y las creencias hacia la maternidad en las mujeres como variable de salida.

\section{Resultados}

Con la información obtenida primero se realizó una prueba $t$ de Student en empoderamiento, autoconcepto y creencias sobre la maternidad para identificar si existen diferencias entre mujeres que planean ser madres y quienes no. Los resultados que se observan en la Tabla 1, muestran de manera general que no existen diferencias, sin embargo, es necesario resaltar las diferencias que se encuentran en torno a las creencias hacia la maternidad como sentido de vida $(\mathrm{t}(231)=2.28$, sig=0.02)

Tabla 1.

Diferencia en la percepción de empoderamiento y autoconcepto en mujeres que planean tener hijas o hijos con quienes no planean

\begin{tabular}{|c|c|c|c|c|c|c|c|c|}
\hline & & \multicolumn{2}{|c|}{$\begin{array}{c}\text { Planean } \\
\text { tener hijos/ } \\
\text { as }(n=191)\end{array}$} & \multicolumn{2}{|c|}{$\begin{array}{l}\text { No planean } \\
\text { tener hijos/ } \\
\text { as }(n=46)\end{array}$} & \multirow[t]{2}{*}{$\mathrm{t}$} & \multirow[t]{2}{*}{ gl } & \multirow[t]{2}{*}{ sig. } \\
\hline & & M & SD & M & SD & & & \\
\hline \multirow{2}{*}{$\begin{array}{l}\text { Creencias hacia la } \\
\text { maternidad }\end{array}$} & Sentido de vida & 14.15 & 5.79 & 11.91 & 6.49 & 2.28 & 231 & 0.02 \\
\hline & Deber social & 4.91 & 1.84 & 4.71 & 2.02 & 0.65 & 229 & 0.51 \\
\hline \multirow[t]{2}{*}{ Empoderamiento } & $\begin{array}{l}\text { Baja agencia } \\
\text { personal }\end{array}$ & 43.46 & 7.28 & 42.66 & 7.55 & 0.62 & 218 & 0.53 \\
\hline & Empoderamiento & 15.73 & 4.09 & 16.28 & 4.28 & -0.81 & 234 & 0.41 \\
\hline \multirow[t]{9}{*}{ Autoconcepto } & Social expresivo & 25.72 & 3.14 & 25.77 & 3.12 & -0.10 & 232 & 0.91 \\
\hline & Ético normativo & 18.28 & 1.68 & 18.22 & 1.96 & 0.20 & 230 & 0.83 \\
\hline & $\begin{array}{l}\text { Inteligencia } \\
\text { socioemocional }\end{array}$ & 19.12 & 3.35 & 17.46 & 3.77 & 2.80 & 230 & 0.005 \\
\hline & $\begin{array}{l}\text { Control externo } \\
\text { negativo pasivo }\end{array}$ & 9.58 & 2.96 & 10.53 & 3.65 & -1.82 & 227 & 0.06 \\
\hline & Social afiliativo & 20.31 & 3.23 & 18.82 & 3.75 & 2.70 & 229 & 0.007 \\
\hline & $\begin{array}{l}\text { Emotivo negativo } \\
\text { auto afirmativo }\end{array}$ & 11.20 & 2.95 & 11.82 & 2.89 & -1.28 & 230 & 0.20 \\
\hline & $\begin{array}{l}\text { Instrumental } \\
\text { constructivo }\end{array}$ & 16.44 & 2.17 & 16.50 & 2.22 & -0.16 & 234 & 0.87 \\
\hline & $\begin{array}{l}\text { Vulnerabilidad } \\
\text { emocional }\end{array}$ & 12.12 & 3.37 & 12.75 & 3.36 & -1.11 & 229 & 0.26 \\
\hline & Depresivo & 11.89 & 3.07 & 12.04 & 2.91 & -0.28 & 227 & 0.77 \\
\hline
\end{tabular}


en donde se reporta que las mujeres que planean tener hijas/hijos tienen una media mayor ( $\mathrm{M}=14.15, \mathrm{SD}=5.79)$ en comparación con aquellas mujeres que no lo planean $(\mathrm{M}=11.91, \mathrm{SD}=6.49)$. Por otro lado, en la dimensión de autoconcepto se identifican diferencias significativas en donde las mujeres que planean tener hijos/hijos reportan tener mayor media en inteligencia socioemocional ( $t(230)=2.80$, sig. $=0.005)$ y características social afiliativas $(\mathrm{t}(229)=2.70$, sig. $=0.007)$ en comparación con las mujeres que reportan no planear tener hijos.

Posteriormente, se llevó a cabo una t de Student entre mujeres que son madres y quienes no lo son en el momento de la aplicación. Los resultados que se muestran en la Tabla 2 indican que existe una diferencia en el empoderamiento $(t(292)=0.06$, sig. $=0.001)$ en donde las mujeres que son madres reportan tener mayor media en comparación con aquellas quienes nos son madres; es decir las madres perciben que tienen mayor incidencia en su contexto. También se encontró una diferencia en la dimensión de autoconcepto de percepción instrumental constructivo $(\mathrm{t}(291)=-0.24$, sig. $=0.01$ ), en donde las mujeres que no son madres reportan un mayor grado de esta dimensión (Media=16.48, DE=2.18), que se refiere a ser activas, cumplidas, ordenadas y trabajadoras en comparación con las mujeres que son madres (Media=16.35, $\mathrm{DE}=2.83$ ). Es importante indicar que no se encontraron diferencias con respecto a las creencias sobre la maternidad entre mujeres que son madres y quienes no.

Tabla 2.

Diferencia en la percepción de empoderamiento y autoconcepto en madres y no madres

\begin{tabular}{|c|c|c|c|c|c|c|c|c|}
\hline & & \multicolumn{2}{|c|}{$\begin{array}{c}\text { Planean } \\
\text { tener hijos/ } \\
\text { as }(n=264)\end{array}$} & \multicolumn{2}{|c|}{$\begin{array}{l}\text { No planean } \\
\text { tener hijos/ } \\
\text { as }(n=39)\end{array}$} & \multirow[t]{2}{*}{$\mathrm{t}$} & \multirow[t]{2}{*}{$\mathrm{gl}$} & \multirow[t]{2}{*}{ sig. } \\
\hline & & M & SD & M & $\mathrm{SD}$ & & & \\
\hline \multirow{2}{*}{$\begin{array}{l}\text { Creencias hacia } \\
\text { la maternidad }\end{array}$} & Sentido de vida & 16.55 & 7.47 & 13.54 & 5.92 & 2.45 & 286 & 0.60 \\
\hline & Deber social & 5.32 & 1.86 & 4.82 & 1.78 & 1.39 & 284 & 0.34 \\
\hline \multirow[t]{2}{*}{ Empoderamiento } & $\begin{array}{l}\text { Baja agencia } \\
\text { personal }\end{array}$ & 39.82 & 7.39 & 43.15 & 7.45 & -2.24 & 36.20 & 0.90 \\
\hline & Empoderamiento & 15.90 & 5.53 & 15.83 & 4.14 & 0.06 & 292 & 0.005 \\
\hline \multirow[t]{9}{*}{ Autoconcepto } & Social expresivo & 26.12 & 2.78 & 25.77 & 3.11 & 0.61 & 294 & 0.32 \\
\hline & Ético normativo & 18.58 & 1.82 & 18.20 & 1.80 & 1.11 & 295 & 0.63 \\
\hline & $\begin{array}{l}\text { Inteligencia } \\
\text { socioemocional }\end{array}$ & 17.51 & 3.28 & 18.83 & 3.49 & -1.98 & 293 & 0.53 \\
\hline & $\begin{array}{l}\text { Control externo } \\
\text { negativo pasivo }\end{array}$ & 9.44 & 2.64 & 9.68 & 2.98 & -0.40 & 294 & 0.83 \\
\hline & Social afiliativo & 21.31 & 3.13 & 19.94 & 3.34 & 2.19 & 271 & 0.90 \\
\hline & $\begin{array}{l}\text { Emotivo negativo } \\
\text { auto afirmativo }\end{array}$ & 11.60 & 3.20 & 11.28 & 2.93 & 0.55 & 4.07 & 0.42 \\
\hline & $\begin{array}{l}\text { Instrumental } \\
\text { constructivo }\end{array}$ & 16.35 & 2.83 & 16.48 & 2.18 & -0.24 & 291 & 0.01 \\
\hline & $\begin{array}{l}\text { Vulnerabilidad } \\
\text { emocional }\end{array}$ & 11.26 & 4.13 & 12.21 & 3.35 & -1.42 & 289 & 0.09 \\
\hline & Depresivo & 11.24 & 3.26 & 11.87 & 3.06 & -1.05 & 288 & 0.73 \\
\hline
\end{tabular}


El siguiente análisis que se llevó a cabo fue la correlación de Pearson entre las variables de estudio, como se muestra en la Tabla 3 se puede observar una correlación significativa entre las creencias sobre la maternidad como deber social y sentido de vida con la baja agencia personal que refiere a inseguridad y falta de confianza, es decir cuando tienen mayor grado de acuerdo con las creencias sobre la maternidad también presentaran mayor inseguridad y falta de confianza. También en la Tabla 3 se muestra una relación entre las creencias de la maternidad como deber social con características de autoconcepto como es control externo negativo pasivo y vulnerabilidad emocional. Finalmente, se encontraron relaciones entre las creencias de la maternidad como sentido de vida con las dimensiones del autoconcepto de ético normativo e instrumental constructiva.

Tabla 3.

Correlación entre las creencias de la maternidad como deber social y sentido de vida con el empoderamiento y el autoconcepto

\begin{tabular}{|c|c|c|c|c|c|}
\hline & & \multicolumn{2}{|c|}{ Deber social } & \multicolumn{2}{|c|}{$\begin{array}{l}\text { Sentido de } \\
\text { vida }\end{array}$} \\
\hline & & $\mathrm{r}$ & sig. & $\mathrm{r}$ & sig. \\
\hline \multirow[t]{2}{*}{ Empoderamiento } & Baja agencia personal & .280 & .000 & .248 & .000 \\
\hline & Empoderamiento & -.025 & .678 & -.069 & .239 \\
\hline \multirow[t]{9}{*}{ Autoconcepto } & Social expresivo & -.092 & .121 & -.086 & .143 \\
\hline & Ético normativo & -.072 & .225 & -.119 & .044 \\
\hline & Inteligencia socioemocional & .014 & .813 & .001 & .990 \\
\hline & Control externo negativo pasivo & .262 & .000 & .097 & .104 \\
\hline & Social afiliativo & -.013 & .820 & -.028 & .635 \\
\hline & Emotivo negativo auto afirmativo & .050 & .403 & .011 & .853 \\
\hline & Instrumental constructivo & -.086 & .145 & -.141 & .015 \\
\hline & Vulnerabilidad emocional & .150 & .011 & .053 & .368 \\
\hline & Depresivo & .124 & .037 & .003 & .960 \\
\hline
\end{tabular}

Finalmente, se realizaron los análisis de regresión y se muestran los resultados en las Tablas 4 y 5. En la Tabla 4 se puede observar la influencia del empoderamiento y autoconcepto en las creencias de maternidad como deber social; este modelo explica el $8 \%$ de la varianza y es explicada principalmente por el empoderamiento con baja agencia personal (Beta: 0.16) y depresión (Beta: -0.17). Estos resultados indican que entre mayor depresión (melancólica, nerviosa, ansiosa y triste), menor es la percepción de la maternidad como un deber social; mientras que a mayor baja agencia personal como desconfianza y baja seguridad en sí mismas se tendrá existe una mayor tendencia a percibir a la maternidad como un deber social. 
Tabla 4.

Efecto de empoderamiento y autoconcepto sobre creencias de maternidad como deber social

\begin{tabular}{|c|c|c|c|c|c|c|}
\hline & Factores & Beta & B & $\begin{array}{l}\text { Std. } \\
\text { Error } \\
\text { B }\end{array}$ & $95 \% \mathrm{IC}$ & sig. \\
\hline \multirow[t]{2}{*}{ Empoderamiento } & Baja agencia personal & 0.16 & 0.02 & 0.01 & {$[0.00,0.04]$} & 0.04 \\
\hline & Empoderamiento & 0.06 & 0.01 & 0.01 & {$[-0.01,0.05]$} & 0.32 \\
\hline \multirow[t]{9}{*}{ Autoconcepto } & Social expresivo & -0.03 & -0.01 & 0.03 & {$[-0.07,0.04]$} & 0.63 \\
\hline & Ético normativo & -0.07 & -0.04 & 0.05 & {$[-0.14,0.04]$} & 0.32 \\
\hline & $\begin{array}{l}\text { Inteligencia } \\
\text { socioemocional }\end{array}$ & -0.00 & -0.00 & 0.02 & {$[-0.05,0.04]$} & 0.90 \\
\hline & $\begin{array}{l}\text { Control externo negativo } \\
\text { pasivo }\end{array}$ & 0.13 & 0.05 & 0.03 & {$[-0.01,0.12]$} & 0.10 \\
\hline & Social afiliativo & 0.04 & 0.01 & 0.02 & {$[-0.03,0.06]$} & 0.52 \\
\hline & $\begin{array}{l}\text { Emotivo negativo auto } \\
\text { afirmativo }\end{array}$ & -0.03 & -0.01 & 0.03 & {$[-0.07,0.04]$} & 0.66 \\
\hline & $\begin{array}{l}\text { Instrumental } \\
\text { constructivo }\end{array}$ & 0.01 & 0.00 & 0.03 & {$[-0.06,0.08]$} & 0.88 \\
\hline & $\begin{array}{l}\text { Vulnerabilidad } \\
\text { emocional }\end{array}$ & 0.08 & 0.2 & 0.03 & {$[-0.03,0.09]$} & 0.39 \\
\hline & Depresivo & -0.17 & -0.06 & 0.03 & {$[-0.12,-0.00]$} & 0.03 \\
\hline
\end{tabular}

Tabla 5.

Efecto de empoderamiento y autoconcepto sobre creencias de maternidad como sentido de vida

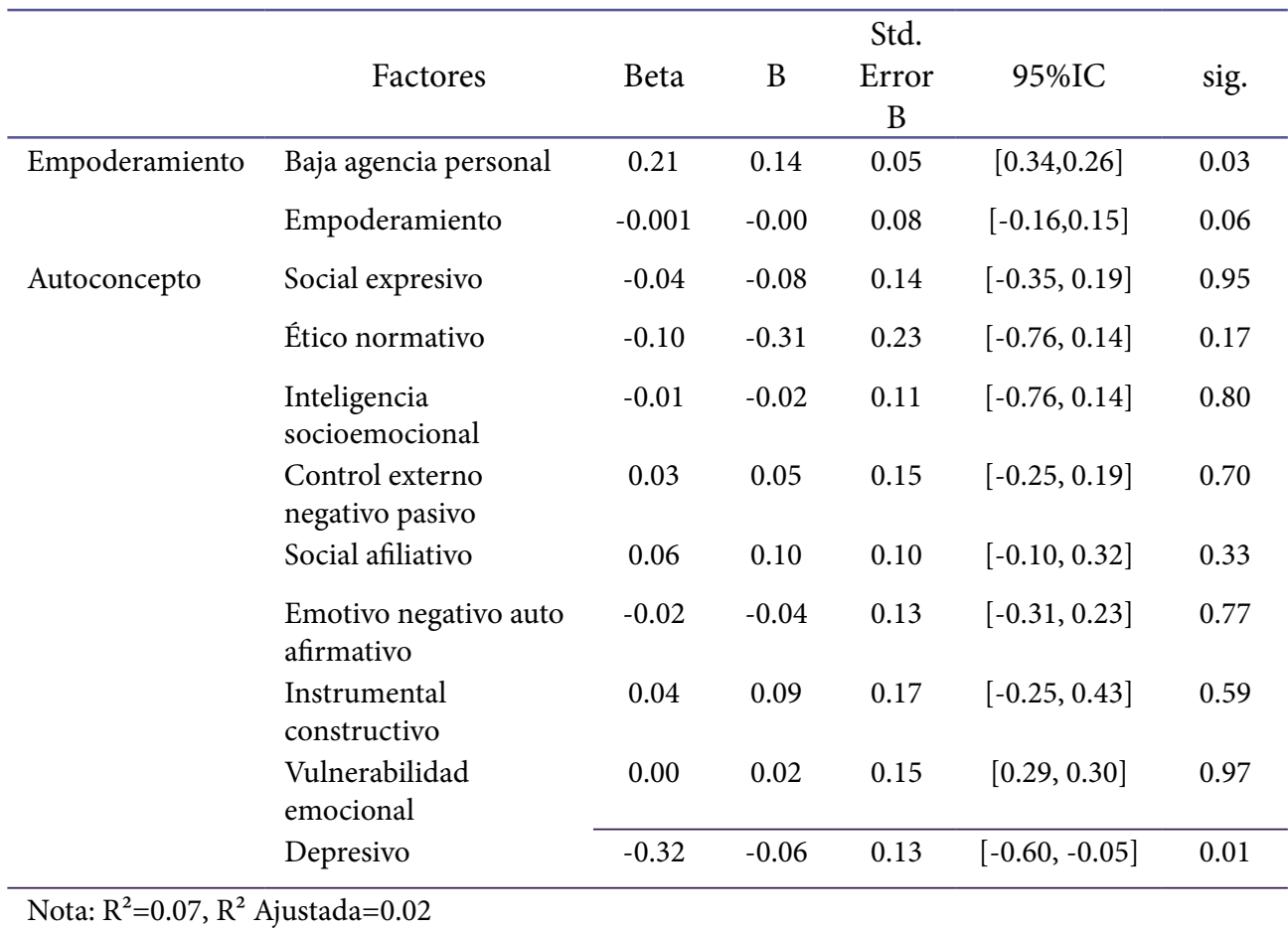


La Tabla 5 presentan los resultados de la regresión del efecto de empoderamiento y autoconcepto sobre creencias de maternidad como sentido de vida. Este modelo explica el $7 \%$ de la varianza y esta explicado principalmente la dimensión de baja agencia personal (Beta de 0.16) y la depresión en la dimensión del autoconcepto (Beta de -0.17), lo cual parece ser un patrón similar con las creencias de la maternidad como deber social. Podemos observar con la beta que entre menos depresión (melancólica, nerviosa, ansiosa y triste), mayor es la percepción de la maternidad como un sentido de vida. Igualmente, podemos ver que a cuando aumenta la baja agencia personal (desconfianza y falta de seguridad en sí mismas) existe una mayor tendencia a percibir a la maternidad como un sentido de vida.

\section{Discusión}

El objetivo de este estudio consistió en identificar la posible influencia que tiene la percepción de empoderamiento y autoconcepto en las creencias que tienen las mujeres sobre la maternidad; los hallazgos de esta investigación permiten identificar que efectivamente existe un efecto. En donde la baja agencia personal, así como la depresión que perciban de sí mismas a partir de elementos de nerviosismo, ansiedad, tristeza y melancolía permean en el aumento de las creencias de la maternidad como deber social y sentido de vida. Estas actitudes favorables hacia las creencias sobre la maternidad refieren ideas en las que se resalta el papel de las mujeres como madres, y que se alcanza la felicidad a partir de este rol, asimismo, consideraran que el valor de la mujer incide en su papel como madre. Es posible entonces identificar que esta idea tradicional sobre el papel de las mujeres a través de la maternidad se mantiene actualmente como lo indica Langer (2002). Particularmente, se encuentran actitudes que contextualmente en México han sido señaladas por Rocha y Díaz Loving (2005) como los roles esperados socialmente como mujeres y en las que se enfatiza el rol de la maternidad como aquel elemento que genera felicidad y propósito a las mujeres (Mota et al., 2018). Es interesante identificar que los estudios anteriores marcaban un contexto social sobre la importancia da la maternidad en contraste, con este estudio es posible identificar que estas creencias sobre la maternidad aumentan cuando las mujeres reportan tener altos niveles de depresión y una baja agencia, lo cual, provee una explicación sobre la finalidad que identifican en la maternidad como aquel rol que proveerá mayores satisfacciones y la cual, deben de logar de acuerdo con Valverde y Cubero (2014).

De igual manera, esta investigación provee con información en la que se muestra que la vinculación de las actitudes hacia la maternidad se encuentra en distintas áreas del autoconcepto. Cuando las mujeres consideran a la maternidad como un deber social en donde es enfatiza el valor de la mujer cuando es madre, se observan que ellas se perciben con características que socialmente no son valoradas desde una mirada tradicional de las mujeres abnegadas (Rocha y Díaz Loving, 2005) ya que, ellas se consideran chantajistas, manipuladoras, apáticas, tímidas, así como 
refieren encontrares en soledad y vulnerabilidad que son rechazadas socialmente y cumpliendo los estereotipos de las mujeres quienes no son madres como refiere Danilu (1999). Por lo tanto, es posible que se identifique un cambio sobre su valoración social cuando tomen un nuevo rol, y por ende un cambio en su autoconcepto que sea congruente con las expectativas que se generan en el marco de la maternidad que refiera a habilidades para formar relaciones sociales como refiere Ávila (2005) y Castillo (2008).

Asimismo, en los contextos sociales se ha vinculado a la maternidad con características de madurez cognitiva y emocional (Daniluk, 1999) a partir de esta vinculación es posible identificar esta relación que se encontró en esta investigación en referencia de las actitudes hacia la maternidad como sentido de vida con aspectos personales del autoconcepto como características de cumplimiento, trabajadoras, ordenadas y activas, así como aquellas características que corresponden a la dimensión de ética normativa en la que se enfatiza la honestidad, honradez, lealtad y respeto. Vinculando las dimensiones culturales que nos enmarca las expectativas sobre las mujeres quienes son madres con las características personales como un elemento que conlleva a la maternidad obligatoria por contar con las habilidades requisitadas socialmente.

Esta investigación muestra que las diferencias con respecto a las actitudes hacia la maternidad se encuentran en mujeres quienes desean ser madres por primera vez o subsecuente en comparación con aquellas mujeres quienes no desean tener otro o ningún otra hija o hijo. Estas diferencias se encuentran en las actitudes hacia la maternidad ya que, cuando las mujeres desean la maternidad considerarán que esta maternidad es proveedora de sentido de vida y valoración de las mujeres como lo indica Valverde y Cubero (2014). Asimismo, es posible identificar que aquellas mujeres quienes desean ser madres o son madres se consideran como tranquilas, serenas, calmadas, y relajadas socialmente, así como tiernas, sentimenteras, amorosas y cariñosas en sus interacciones sociales. Un elemento en el cual se identificaron diferencias es en el empoderamiento en donde las mujeres quienes son madres nos refieren ser participativa en su comunidad y ayuda a resolver conflictos que se presentan, es decir, se refiere a una mujer que tiene incidencia en su comunidad en comparación con aquellas mujeres quienes son madres. Estos hallazgos nos cuestionan el lugar de la incidencia y provee un elemento sobre el impacto social que tienen las actividades de cuidado que se realizan desde el rol de la maternidad, como se ha evidenciado en distintas investigaciones sobre la economía del cuidado (INMUJERES, 2011).

A partir de esta investigación, se identifica que las nociones tradicionales sobre la maternidad pueden tener una relación con los elementos de autoconcepto y, por ende, en las actitudes hacia la maternidad. En la literatura existe evidencia de la influencia sociocultural en las construcciones individuales, como en este caso, en el autoconcepto (Al-Lal, Herrera, y Mohamed, 2017; Esteban, Bastiani y Vila, 2009).

Finalmente, esta investigación permite comprender la percepción, y creencias que 
tienen las mujeres mexicanas actualmente ante la maternidad y su vinculación con el empoderamiento y el autoconcepto. Por lo que se generan nuevas preguntas sobre la reinterpretación que se tiene a partir de un autoconcepto que no es socialmente reconocido y su vinculación con su deseo de ser madres, la cual está asociado con su creencia de la maternidad como un valor social. También es necesario cuestionarse el cambio que se busca tener sobre la imagen social de la maternidad porque una de las riquezas que se ha identificado y que requiere visibilidad es su incidencia social en su comunidad que se ha integrado como un elemento de empoderamiento pero que puede tener un impacto favorable en la economía social y político.

\section{Referencias}

Al-Lal, M., Herrera, T. L. y Mohamed, M. L. (2017). Personalidad y autoconcepto según el origen cultural de estudiantes de educación primaria. La psicología hoy: Retos, logros y perspectivas de futuro. Psicología infantil, 1(2), 295-304.

Anzaldúa, G. (2014). Los movimientos de rebeldía y las culturas que traicionan. En B. Hooks, A. Brah, C. Sandoval, G. Anzaldúa, A. Levins Morales, K.-K. Bhavnani, M. Coulson, J. Alexander y C. Talpade Mohanty (Eds.), Otras inapropiables: Feminismos desde las fronteras (pp. 71-81). Traficantes de Sueños.

Ávila, G. (2005). Mujeres frente a los espejos de la maternidad: Las que eligen no ser madres. Desacatos, 17, 107-126.

Bethencourt, L. y Carrillo, M. (2014). Mujeres, empoderamiento y transformaciones socioeconómicas y políticas. Caso municipio Valdez, estado Sucre. Cuadernos del CENDES, 31(85), 157-161.

Burns, R. (1979). The self-concept. Theory, measurement, development and behavior. Longman.

Castillo, M. (2008). Modelos y prácticas de maternidad: Continuidades y cambios en dos generaciones de madres platenses. Revista Mad, 19, 63-79.

Daniluk, J. (1999). When biology isn't destiny: Implications for sexuality of women without children. Canadian Journal of Counselling, 32(2), 79-94.

Esnaola, I., Goñi, A. y Madariaga, J. (2008). El autoconcepto: Perspectivas de investigación. Revista de Psicodidáctica, 13(1), 69-96.

Esteban, M., Batiani, J. y Vila, I. (2009). El impacto de la cultura en el autoconcepto. Un estudio con mestizos de distintos entornos educativos de Chiapas. Infancia y Aprendizaje, 21(3), 361-370.
Fundación Nacional por la Familia. (2019). Quiénes somos. HTTP://FRENTENACIONAL.MX/ QUIENESSOMOS/

González, C., Calleja, N., Bravo, C. y Meléndez, J. (2019). Escala de creencias sobre la maternidad: Construcción y validación en mujeres mexicanas. Revista Iberoamericana de Diagnóstico y Evaluación - e Avaliação Psicológica, 50(4), 163172. HTTPS://DOI.ORG/10.21865/RIDEP50.1.13

González, M. y Touron, J. (1992). Autoconcepto $y$ rendimiento escolar. Sus implicaciones en la motivación de la autorregulación del aprendizaje. EUNSA.

González, O. y Goñi, E. (2005). Dimensiones del autoconcepto social. International Journal of Developmental and Educational Psychology, 2(1), 249-261.

Harter, S. (1990). Developmental differences in the nature of self-representations: Implications for the understanding, assessment, and treatment of maladaptive behavior. Cognitive Therapy and Research, 14, 113-142. HTTPS://DOI.ORG/10.1007/ BF01176205

INEGI. (2019). Natalidad y fecundidad. нTTPs:// WWW.INEGI.ORG.MX/TEMAS/NATALIDAD/

Instituto Nacional de las Mujeres. (2011). Trabajo doméstico no remunerado equivale a $21.7 \%$ del PIB. Comunicado de prensa 61. WWW.INMUJERES. GOB.MX/SALA-DE-PRENSA

Instituto Nacional de las Mujeres. (2018). Las madres en cifras. HTTPS://WWW.GOB.MX/INMUJERES/ ARTICULOS/LAS-MADRES-EN-CIFRAS

Instituto Nacional de las Mujeres. (2019). Proequidad (igualdad de género). нтtps://WwW.Goв. MX/INMUJERES/ACCIONES-Y-PROGRAMAS/ PROGRAMA-PROEQUIDAD-IGUALDAD-DE-GENERO 
Kimble, C., Hirt, E., Díaz-Loving, R., Hosch, H., Lucker, W. y Zárate, M. (2002). Psicología social de las américas. Prentice Hall.

Langer, A. (2002). El embarazo no deseado: Impacto sobre la salud y la sociedad en América Latina y el Caribe. Revista Panamericana de Salud Pública, 11(3), 192-203.

Loeber, R. (1986). Family factors as correlates and predictors of juvenile conduct problems and deliquency. Crime and Justice, 7, 29-149.

OMS. (2016). Salud reproductiva. нттрs://WwW. WHO.INT/TOPICS/REPRODUCTIVE_HEALTH/ES/

Pérez-Duarte, A. E. (1996). La maternidad: Relato de una contradicción. En Ética y salud reproductiva. Porrúa-UNAM.

Pick, S., Sirkin, J., Ortega, I., Osorio, P., Martínez, R., Xocolotzin, U. y Givaudan, M. (2007). Escala Para Medir Agencia Personal y Empoderamiento (ESAGE). Interamerican Journal of Psychology, 41(3), 295-304.

Rasanen, P., Hakko, H., Isohanni, M., Hodgins, S., Jarvelin, M. y Tiihonen, J. (1999). Maternal smoking during pregnancy and risk of criminal behavior among adult male offspring in the Northern Finland 1966 Birth Cohort. Am J Psychiatry, 156, 857-862.

Reyes-Lagunes, I., Rivera-Aragón, S. y Díaz-Loving, R. (2002). Autoconcepto: Desarrollo y validación de un inventario etnopsicológico. Revista Iberoamericana de Evaluación Psicológica, 13(1), 29-54.
Rodríguez, C. (2018). Empoderamiento de mujeres rurales del sur de Cauca: Un caso regional de innovación social [Tésis de máster inédita]. Universidad ICESI

Sampson, R. y Laub, J. (1993). Crime in the making: Pathways and turning points through life. Harvard University Press.

Sánchez, B. N. (2016). La experiencia de la maternidad en mujeres feministas. Nómadas, 44, 255-267.

Shavelson, R. J., Hubner, J. J. y Stanton, G. C. (1976). Self-concept: Validation of construct interpretations. Review of Educational Research, 46(3), 407-441. HTTPs://DOI. ORG/10.3102/00346543046003407

Trujano, R. (2006). Paternidad, maternidad, y empoderamiento femenino. Revista Electrónica de Psicología Iztacala, 9(3), 86-108.

Valverde, K. y Cubero, M. (2014). La maternidad como un constructo social determinante en el rol de la feminidad. Revista Wimb Lu, 9(1), 29-42.

Vivas, E. (2019). Mamá desobediente. Una mirada feminista a la maternidad. Capitan Swing.

Zabala, F. y Fernández, R. (2005). Diferencias en el autoconcepto personal y social. International Journal of Developmental and Educational Psychology, 1(1), 243-256. 